\title{
An Update on the Emerging Role of Visfatin in the Pathogenesis of Osteoarthritis and Pharmacological Intervention
}

\author{
Dong-Feng Han, ${ }^{1}$ Yang $\mathrm{Li}^{2}{ }^{2}$ Hui-Ying $\mathrm{Xu},{ }^{3}$ Rong-Hang $\mathrm{Li},{ }^{4}$ and Ding Zhao $\mathbb{D}^{5}$ \\ ${ }^{1}$ Department of Emergency Medicine, The First Hospital of Jilin University, Changchun, Jilin 130021, China \\ ${ }^{2}$ Department of Thoracic Surgery, The First Hospital of Jilin University, Changchun, Jilin 130021, China \\ ${ }^{3}$ Department of Ultrasound, The First Hospital of Jilin University, Changchun, Jilin 130021, China \\ ${ }^{4}$ Department of Orthopedic Surgery, The Second Hospital of Jilin University, Changchun, Jilin 130021, China \\ ${ }^{5}$ Department of Orthopedic Surgery, The First Hospital of Jilin University, Changchun, Jilin 130021, China \\ Correspondence should be addressed to Ding Zhao; zhaoding1983@163.com
}

Received 6 April 2020; Revised 10 June 2020; Accepted 18 June 2020; Published 8 August 2020

Academic Editor: Youn-Chul Kim

Copyright (c) 2020 Dong-Feng Han et al. This is an open access article distributed under the Creative Commons Attribution License, which permits unrestricted use, distribution, and reproduction in any medium, provided the original work is properly cited.

\begin{abstract}
Osteoarthritis (OA) is one of the most common degenerative joint diseases that affects millions of people worldwide, mainly the aging population. Despite numerous published reports, little is known about the pathology of this disease, and no feasible treatment plan exists to stop OA progression. Recently, extensive basic and clinical studies have shown that adipokines play a key role in OA development. Moreover, some drugs associated with adipokines have shown chondroprotective and anti-inflammatory effects on OA. Visfatin has been shown to play a detrimental role in the progression of OA. It increases the production of matrix metalloproteinases and a disintegrin and metalloproteinase with thrombospondin motifs (ADAMTS), induces the production of interleukin (IL)- $1 \beta$, IL-6, and tumor necrosis factor- $\alpha$, affects the differentiation of mesenchymal stem cells to adipocytes, and induces osteophyte formation by inhibiting osteoclastogenesis. Although some side effects of chemical visfatin inhibitors have been reported, they were shown to be successful in the treatment of diabetes, cancer, and other diseases that can utilize Chinese herbs, further suggesting that similar therapeutic strategies could be used in OA prevention and treatment. Here, we describe the pathophysiological mechanism of visfatin in OA and discuss some potential pharmacological interventions using Chinese herbs.
\end{abstract}

\section{Introduction}

Osteoarthritis (OA) is one of the most common forms of arthritis and a major cause of disability in the elderly population $[1,2]$. In the past, OA was generally attributed to mechanical processes, known as the "wear and tear" paradigm [3]. However, numerous cohort studies have reported that obesity is also a risk factor for OA in non-weight-bearing joints $[4,5]$. In recent years, some studies have shown that adipokines, secreted by white adipose tissue (WAT), also play important roles in OA. For example, leptin and resistin induce the production of prostaglandin E (PGE), matrix metalloprotein (MMP) -1 and -13, and other inflammatory factors. On the contrary, adiponectin plays a protective role in $\mathrm{OA}$ by inhibiting the release of MMP-13 and upregulating the expression of type II and type $\mathrm{X}$ collagens [3, 6-9].
Adipokines have a systemic effect on the endocrine system and may be a vital link between obesity and OA [10]. Recently, there has been great interest in the role of the fat cell-derived protein, visfatin, in the pathophysiology of OA because it plays a crucial role in cartilage and bone homeostasis $[11,12]$. The purpose of this review is to summarize the current knowledge on the pathophysiological mechanism of visfatin in $\mathrm{OA}$ and discuss potential pharmacological interventions.

\section{Visfatin}

Visfatin, discovered in 1994, is a $52 \mathrm{kD}$ adipokine protein, also known as pre-B cell colony-enhancing factor (PBEF) and nicotinamide phosphoribosyltransferase (NAMPT) [13]. Visfatin promotes B cell maturation and is expressed 
widely in WAT and other tissues of humans $[14,15]$. Haider et al. compared visfatin levels in lean and obese individuals and the effect of exercise training on its concentrations. They found that serum levels of visfatin increase in obese individuals and can be reduced by losing weight [16]. Although some researchers proved that visfatin shows the function of insulin-mimetic, the role of visfatin in glucose metabolism is still unclear [17-19]. Other studies show that visfatin induces chemotaxis and production of interleukins (IL) -1 and -6 , as well as tumor necrosis factor- $\alpha$ (TNF- $\alpha$ ) in lymphocytes of obese patients. This suggests that visfatin may be involved in obesity-associated inflammatory states [20].

\section{Visfatin in OA Patients}

In recent years, several researchers have measured visfatin levels in patients with OA. Some researchers revealed that circulating and local visfatin levels in these patients were higher than those in healthy controls (serum concentration: 36.3 vs. $27.3 \mathrm{ng} / \mathrm{ml}, p<0.05$; synovial fluid (SF): 8.95 vs. $4.48 \mathrm{ng} / \mathrm{ml}, p<0.001$ ) [12]. These results indicate that visfatin is involved in the pathophysiological process of OA, and articular tissues may affect the SF levels of visfatin [21].

Interestingly, cartilage and synovial tissues of patients with OA have been shown to secrete more visfatin than those of healthy subjects [22]. Moreover, the expression of visfatin in infrapatellar fat pad tissues of OA patients is higher than that in matched subcutaneous WAT [23]. Furthermore, visfatin has also been shown to be expressed in osteophytes by various articular cell types including osteoblasts, osteoclasts, and chondrocytes in patients with OA [24].

Levels of visfatin in serum or SF appear to be associated with lipid metabolism, inflammation, and progression of clinical disease [25]. Lee and Bae analyzed serum visfatin and C-reactive protein (an inflammatory marker) levels of 813 patients with rheumatoid arthritis and found a positive correlation. These results indicate that visfatin might be related to lipid metabolism and the inflammatory process [26].

Visfatin levels in SF are increased in patients with OA who show more radiographic evidence of joint damage compared to those with less disease severity. Specifically, Duan et al. reported that SF visfatin levels in K-L grade 4 are significantly elevated compared to those of K-L grade 3 (10.57 vs. $7.54 \mathrm{ng} / \mathrm{ml}, p=0.001$ ) [12].

\section{Roles of Visfatin in OA}

Visfatin plays a detrimental role in extracellular matrix homeostasis [27]. Junker et al. revealed that collagen types II and $\mathrm{X}$ are significantly reduced in chondrocytes that are stimulated by visfatin for $24 \mathrm{~h}$ [24]. Furthermore, visfatin increases the production of matrix metalloproteinases (MMPs) and a disintegrin and metalloproteinase with thrombospondin motifs (ADAMTS). Gosset et al. reported that visfatin-treated mouse articular chondrocytes show increased expression of MMP-3, -13 and ADAMTS-4, -5 [22]. Yang et al. revealed that visfatin increases the mRNA levels and activities of MMP-3, -12, and -13 in human OA cartilage chondrocytes. They further reported that injecting visfatin into the knee joints of mice triggers cartilage destruction by increasing the production of cysteine proteases as well as MMP-3, -12, and -13 in cartilage tissue at both the gene and protein levels [28].

Visfatin exerts a proinflammatory effect during the progression of $\mathrm{OA}$. As discussed earlier, visfatin induces the production of IL-1 $\beta,-6$, and TNF- $\alpha$ in lymphocytes [20]. Amy et al. showed that visfatin enhances the biological effects of IL-1 by increasing the activity of MMPs, nitric oxide (NO) production, and proteoglycan release in cartilage and meniscus tissue [29].

Visfatin affects the differentiation of mesenchymal stem cells (MSCs) and the activity of osteoclasts. Li et al. found that MSC lineage fate determination is affected by cell energy metabolism and revealed a possible mechanism for senile osteoporosis, indicating that visfatin may affect MSC differentiation into adipocytes or osteoblasts [30]. Furthermore, Lali et al. found that IL-8 levels are significantly increased by visfatin during MSC differentiation, and these elevated levels induce the differentiation of human bone marrow cells into osteoclasts [31]. Apart from the effects on osteoblast proliferation and collagen synthesis, visfatin also plays a crucial role in osteoclast formation by inhibiting osteoclastogenesis, which indicates its role in osteophyte formation in the context of inflammatory diseases [20,32]. However, contrary to the above findings, Venkateshaiah et al. showed that visfatin promotes osteoclast activity and myeloma cell growth in multiple myeloma owing to its enzymatic activity [33]. The reasons behind these effects are not clear, and the authors suggest that further studies are required to gain better insight.

\section{Signaling Pathways of Visfatin}

Although a visfatin receptor has not been identified, some researchers have shown that it can bind directly to the insulin receptor (IR) to exert biological effects in certain cell types such as human embryonic kidney 293 and A549 lung epithelial cells $[34,35]$. The IR is expressed widely in humans and murine chondrocytes and plays a key role in the pathophysiological process of OA [22]. Furthermore, Huang et al. showed that visfatin binds to $\beta 1$ integrin to induce the expression of stromal cell-derived factor-1 [36].

Despite the absence of an identified visfatin receptor, there are four signaling pathways associated with visfatin (Figure 1). The first visfatin signaling pathway is mediated by IL- 6 and involves STAT-3, HIF-2 $\alpha$, NAMPT, SIRT1, and SIRT6 pathways. It has been reported that IL-6 trans-signaling affects the expression of visfatin, and the expression was mediated by STAT-3 and HIF-2 [37, 38]. Deacetylation of NAMPT by mammalian NAD+-dependent deacetylase, SIRT1, predisposed the protein to secretion by adipocytes. This is evident in a study with NAMPT mutants that reveals the deacetylation of lysine 53 and enhancement of NAMPT activity and secretion by SIRT1 [39]. Hong et al. also reported that visfatin could activate SIRT1. In response to IL$1 \beta$, SIRT1 activates extracellular signal-regulated kinase (ERK) and p38. The SIRT1-ERK complex participates in the 


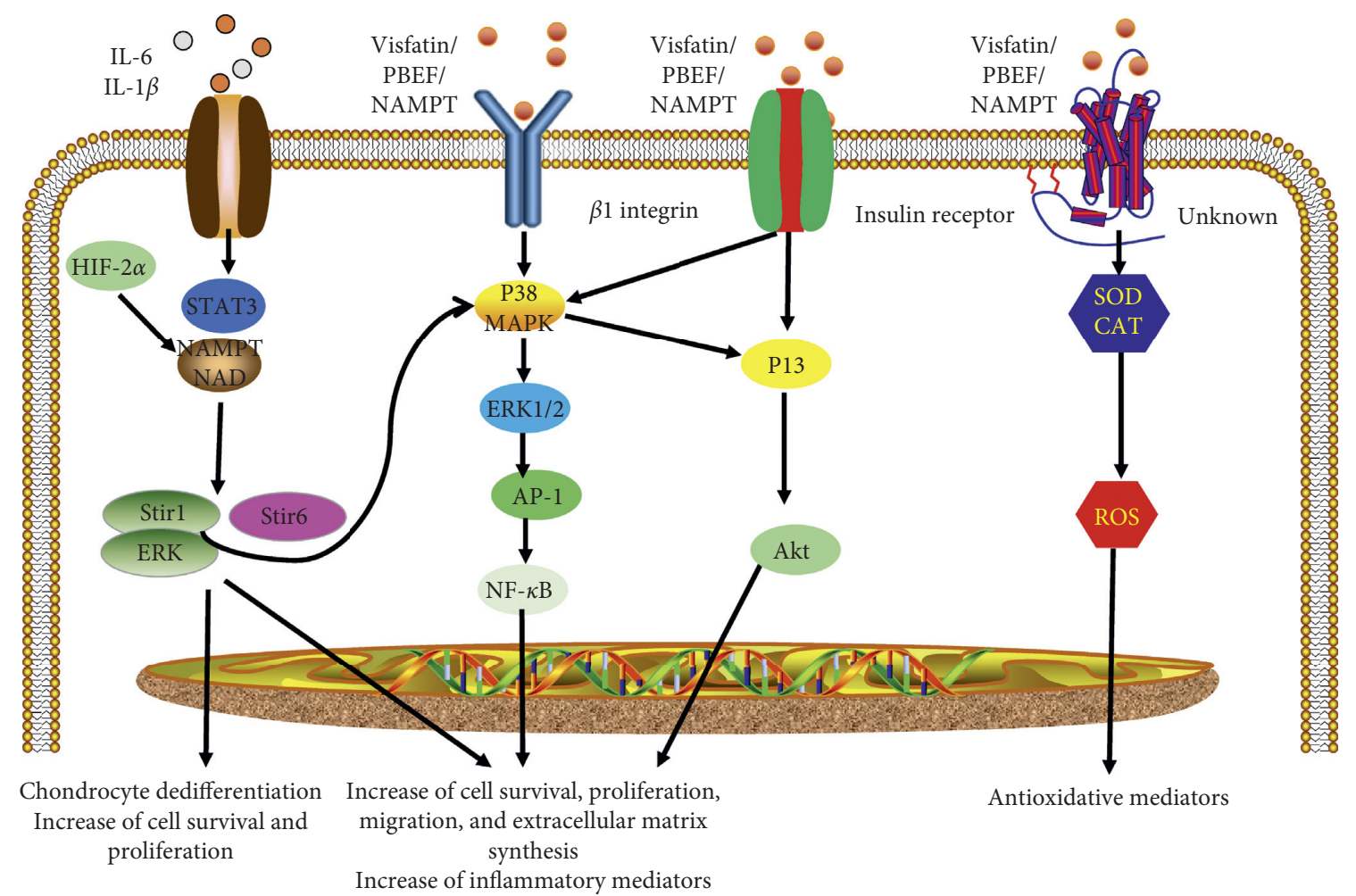

FIGURE 1: Signaling pathways of visfatin. First, visfatin signal is mediated by IL-6 and involves STAT-3, HIF-2 $\alpha$, NAMPT, SIRT1, and SIRT6 pathways. Second, visfatin signal is mediated by $\beta 1$ integrin and involves ERK, p38 mitogen-activated protein kinase (MAPK), nuclear factor $-\kappa \mathrm{B}(\mathrm{NF}-\kappa \mathrm{B})$, and AP-1 pathways. Third, visfatin signal is mediated by the IR and involves the PI3K, Akt, MAPK, and ERK1 pathways. Fourth, visfatin signal is demonstrated through the activation of an unknown receptor increasing the antioxidative enzymes superoxide dismutase (SOD) and catalase (CAT).

dedifferentiation of chondrocytes induced by IL- $1 \beta$ through Sox9-mediated signals. Indeed, visfatin and ERK signaling could strengthen chondrocyte dedifferentiation mediated by SIRT1 [40].

SIRT1 has been shown to have a preventive role in OA by inducing cartilage-specific gene expression and inhibiting chondrocyte apoptosis $[41,42]$. Interestingly, the data also show that visfatin increases NAD+ levels, thereby altering SIRT1 activity. Therefore, SIRT1, visfatin, and NAD+ have positive effects on human cartilage by increasing the expression of genes that encode the cartilage extracellular matrix [42].

The second visfatin signaling pathway is mediated by $\beta 1$ integrin and involves ERK, p38 mitogen-activated protein kinase (MAPK), nuclear factor $-\kappa \mathrm{B}(\mathrm{NF}-\kappa \mathrm{B})$, and $\mathrm{AP}-1$ pathways. Liu et al. reported that visfatin could induce monocyte chemoattractant protein-1 and IL-6 production through p38, MAPK, and phosphoinositide 3-kinase (PI3K) pathways [43]. Moreover, Huang et al. showed that visfatininduced stromal cell-derived factor-1 expression in colorectal cancer cells is mediated via the activation of the $\beta 1$ integrin, ERK/p38, and NF- $\kappa \mathrm{B} / \mathrm{AP}-1$ pathways [36]. Lastly, Raghu et al. reported that visfatin induces the production of MMP-2 and -9 in human endothelial cells via the MAPK and PI3K/Akt signaling [44].

The third visfatin pathway is mediated by the IR and involves the PI3K, Akt, MAPK, and ERK1 pathways. Wang et al. revealed that visfatin stimulates proliferation and promotes the progression of the G1/S phase, as well as suppresses apoptosis in endometrial carcinoma tumor cells through the activation of IR, PI3K/Akt, and MAPK/ERK signaling pathways [45]. Brown et al. also showed that visfatin inhibits pancreatic $\beta$-cell apoptosis through MAPK/ IR/PI3K signaling [46]. Moreover, Claire et al. showed that visfatin regulates the IR pathway activity to induce prostaglandin E2 release in chondrocytes [47].

The fourth signaling pathway involves redox reactions. Visfatin could increase the activity of superoxide dismutase to reduce the production of reactive oxygen metabolites [48]. Based on the protective regulation of this enzyme in cellular redox reactions and energy pathways, visfatin has been used as an effective target for cancer treatment [49].

\section{Pharmacological Interventions}

Visfatin plays an important role in cancer and inflammation; therefore, researchers have shown great interest in developing drugs and exploring traditional Chinese herbs.

Although various visfatin inhibitors have been developed, only three inhibitors (FK866, CHS828, and KPT-9274) have progressed to clinical trials. Although the trial data have not yet been published, some side effects have been reported in phase I/ II clinical trials. These include thrombocytopenia, anemia, gastrointestinal toxicity, and hypoalbuminemia [50]. 
FK866, the most studied visfatin inhibitor, shows a high affinity for visfatin and prevents its biological activity by competing for the same binding site with visfatin [51]. In inflammatory arthritis in mice, the pharmacological inhibition of visfatin by FK866 decreased the severity of arthritis by reducing the expression of MMP-3, -13, and receptor or activator of $\mathrm{NF}-\kappa \mathrm{B}$ ligand (RANKL) in vitro and in vivo [52]. Yang et al. established mouse models of OA by surgical destabilization of the medial menisci of the knee joints of rats or by using an intra-articular injection of visfatin. An intra-articular or intra-abdominal injection of FK866 $(10 \mathrm{mg} / \mathrm{kg})$ significantly inhibits cartilage destruction induced by visfatin and brings about a concomitant reduction of mRNA levels of the visfatin targets MMP-3, -12, and -13 in cartilage tissue [28].

The chemical derivatives of visfatin show a number of side effects; therefore, academic and industrial researchers have started to shift their interest toward traditional Chinese herbs. These outcomes have already been attained in diabetes, oncology, and other diseases using Chinese herbs, which suggest that similar strategies could be used to prevent and treat OA [53-56]. The mechanisms of action of Chinese herbs in regulating the biological effects of visfatin in arthritis require further study.

Curcumin is a natural compound extracted from the rhizome of turmeric that possesses antioxidant, anti-inflammatory, antiatherogenic, and antitumor activities [57]. Li et al. showed that curcumin can improve nonalcoholic fatty liver disease (NAFLD) by reducing the expression of visfatin in NAFLD rats [58]. In some cancer, the same results were found about curcumin which could decrease the expression of visfatin [59]. Furthermore, it suppresses synovial inflammation, oxidative stress, and matrix degradation of inflammatory chondrocytes through the Nrf2/ARE and NF$\kappa \mathrm{B}$ signaling pathways [60]. In clinical trials, curcumin has proven to be effective in treating patients with $\mathrm{OA}$ and improving their Western Ontario and McMaster Universities Osteoarthritis Index (WOMAC) scores [61].

Emodin, an active component of Rheum palmatum and other Chinese herbs including Polygonum cuspidatum, Polygonum multiflorum, and Cassia obtusifolia, has been shown to possess anti-inflammatory, antiatherogenic, and antitumor activities [55]. Cui et al. showed that emodin alleviates severe acute pancreatitis (SAP) that is associated with acute lung injury (ALI) by decreasing visfatin expression and promoting polymorphonuclear neutrophil apoptosis [62]. In OA, emodin could protect cartilage from degradation by suppressing $\mathrm{NF}-\kappa \mathrm{B}$ and $\mathrm{Wnt} / \beta$-catenin signaling, which has been observed in cellular and animal experiments [63].

Genistein, a primary active ingredient of Sophora japonica and Sandougen, is known to inhibit inflammatory responses, reduce oxidative stress-induced damage, and exert anticancer effects [64]. Zhu et al. studied whether genistein protected alveolar epithelial cells from LPS-induced injury and reported that genistein plays a protective effect in lung injury by suppressing the activation of NF- $\kappa \mathrm{B}$ and alleviating the inflammatory response [65]. In OA, genistein suppresses the expression of NF- $\kappa$ B activated by inflammatory cytokines and plays a protective role in preventing condylar cartilage damage in OA in rats [66].

Polydatin, a well-known component obtained from Rhizoma Polygoni Cuspidati, is known to have various pharmacological effects and shows anti-inflammatory, blood lipid-regulating, cholesterol lowering, and antishock properties [67]. Studies by Huang et al. revealed that polydatin can relieve atherosclerosis injury in mice through the downregulation of visfatin and inhibition of visfatin-inducing cholesterol deposits in macrophages [54]. In OA, polydatin protects the cartilage from degeneration by activating chondrocyte autophagic flux via the MAPK and PI3K/Akt signaling pathways. Furthermore, polydatin could inhibit inflammation reactions induced by IL- $1 \beta$ in chondrocytes through the activation of the Nrf2 signaling pathway $[68,69]$.

Additionally, other primary active ingredients of traditional Chinese herbs such as Russelioside B (RB) and salidroside have previously been reported to show antidiabetic, anticancer, anti-inflammatory, antishock, and antihyperlipidemic activities. Thus, these compounds may play an important role in protecting joints by regulating visfatin levels $[53,70]$.

\section{Conclusion}

At present, most evidence shows that visfatin plays a proinflammatory role in OA. The increasing interest in visfatin has gradually led to the uncovering of the intricate adipokine-mediated relationship between WAT and OA. Although many aspects are still unclear, this review highlights the molecular functions and mechanisms of visfatin in $\mathrm{OA}$ and discusses some of the potential pharmacological interventions using Chinese herbs. However, further investigations are necessary to fully understand the role of visfatin in $\mathrm{OA}$.

\section{Conflicts of Interest}

The authors declare that they have no conflicts of interest.

\section{References}

[1] R. F. Loeser, S. R. Goldring, C. R. Scanzello, and M. B. Goldring, "Osteoarthritis: a disease of the joint as an organ," Arthritis \& Rheumatism, vol. 64, no. 6, pp. 1697-1707, 2012.

[2] Y. Zhang, L. Xu, M. C. Nevitt et al., "Comparison of the prevalence of knee osteoarthritis between the elderly Chinese population in Beijing and whites in the United States: the Beijing osteoarthritis study," Arthritis \& Rheumatism, vol. 44, no. 9, pp. 2065-2071, 2001.

[3] A. Courties, J. Sellam, and F. Berenbaum, "Metabolic syndrome-associated osteoarthritis," Current Opinion in Rheumatology, vol. 29, no. 2, pp. 214-222, 2017.

[4] S. Kluzek, J. L. Newton, and N. K. Arden, "Is osteoarthritis a metabolic disorder?” British Medical Bulletin, vol. 115, no. 1, pp. 111-121, 2015.

[5] A. R. Sun, S. K. Panchal, T. Friis et al., "Obesity-associated metabolic syndrome spontaneously induces infiltration of 
pro-inflammatory macrophage in synovium and promotes osteoarthritis," PloS One, vol. 12, no. 8, 2017.

[6] Q. Zhuo, W. Yang, J. Chen, and Y. Wang, "Metabolic syndrome meets osteoarthritis," Nature Reviews Rheumatology, vol. 8, no. 12, pp. 729-737, 2012.

[7] X. Wang, D. Hunter, J. Xu, and C. Ding, "Metabolic triggered inflammation in osteoarthritis," Osteoarthritis and Cartilage, vol. 23, no. 1, pp. 22-30, 2015.

[8] R. A. Puenpatom and T. W. Victor, "Increased prevalence of metabolic syndrome in individuals with osteoarthritis: an analysis of NHANES III data," Postgraduate Medicine, vol. 121, no. 6, pp. 9-20, 2009.

[9] R. Gandhi, F. Razak, P. Tso, J. R. Davey, and N. N. Mahomed, "Asian ethnicity and the prevalence of metabolic syndrome in the osteoarthritic total knee arthroplasty population," The Journal of Arthroplasty, vol. 25, no. 3, pp. 416-419, 2010.

[10] V. Abella, M. Scotece, J. Conde et al., "Adipokines, metabolic syndrome and rheumatic diseases," Journal of Immunology Research, vol. 2014, 2014.

[11] S. Zheng, J. Xu, S. Xu et al., “Association between circulating adipokines, radiographic changes, and knee cartilage volume in patients with knee osteoarthritis," Scandinavian Journal of Rheumatology, vol. 45, no. 3, pp. 224-229, 2016.

[12] Y. Duan, D. Hao, M. Li et al., "Increased synovial fluid visfatin is positively linked to cartilage degradation biomarkers in osteoarthritis," Rheumatology International, vol. 32, no. 4, pp. 985-990, 2012.

[13] D. Azamar-Llamas, G. Hernández-Molina, B. Ramos-Ávalos, and J. Furuzawa-Carballeda, "Adipokine contribution to the pathogenesis of osteoarthritis," Rheumatology International, vol. 2017, 2017.

[14] M.-K. Kim, J. H. Lee, H. Kim et al., "Crystal structure of visfatin/pre-B cell colony-enhancing factor $1 /$ nicotinamide phosphoribosyltransferase, free and in complex with the anticancer agent FK-866," Journal of Molecular Biology, vol. 362, no. 1, pp. 66-77, 2006.

[15] B. Samal, Y. Sun, G. Stearns, C. Xie, S. Suggs, and I. McNiece, "Cloning and characterization of the cDNA encoding a novel human pre-B-cell colony-enhancing factor," Molecular and Cellular Biology, vol. 14, no. 2, pp. 1431-1437, 1994.

[16] D. G. Haider, J. Pleiner, M. Francesconi, G. F. Wiesinger, M. Müller, and M. Wolzt, "Exercise training lowers plasma visfatin concentrations in patients with type 1 diabetes," The Journal of Clinical Endocrinology \& Metabolism, vol. 91, no. 11, pp. 4702-4704, 2006.

[17] N. Klöting and I. Klöting, "Visfatin: gene expression in isolated adipocytes and sequence analysis in obese WOKW rats compared with lean control rats," Biochemical and Biophysical Research Communications, vol. 332, no. 4, pp. 1070-1072, 2005.

[18] A. Pfützner and T. Forst, "The release of the adipocytokine visfatin is regulated by glucose and insulin," Diabetologia, vol. 49, no. 11, p. 2795, 2006.

[19] D. G. Haider, K. Schindler, G. Schaller, G. Prager, M. Wolzt, and B. Ludvik, "Increased plasma visfatin concentrations in morbidly obese subjects are reduced after gastric banding," The Journal of Clinical Endocrinology \& Metabolism, vol. 91, no. 4, pp. 1578-1581, 2006.

[20] A. R. Moschen, A. Kaser, B. Enrich et al., "Visfatin, an adipocytokine with proinflammatory and immunomodulating properties," The Journal of Immunology, vol. 178, no. 3, pp. 1748-1758, 2007.

[21] W. P. Chen, J. P. Bao, J. Feng, P. F. Hu, Z. L. Shi, and L. D. Wu, "Increased serum concentrations of visfatin and its production by different joint tissues in patients with osteoarthritis," Clinical Chemistry and Laboratory Medicine, vol. 8, pp. 1141-1145, 2010.

[22] M. Gosset, F. Berenbaum, C. Salvat et al., "Crucial role of visfatin/pre-B cell colony-enhancing factor in matrix degradation and prostaglandin E2 synthesis in chondrocytes: possible influence on osteoarthritis," Arthritis \& Rheumatism, vol. 58, no. 5, pp. 1399-1409, 2008.

[23] J. Conde, M. Scotece, V. Abella et al., "Differential expression of adipokines in infrapatellar fat pad (IPFP) and synovium of osteoarthritis patients and healthy individuals," Annals of the Rheumatic Diseases, vol. 73, no. 3, pp. 631-633, 2014.

[24] S. Junker, K. W. Frommer, G. Krumbholz et al., "Expression of adipokines in osteoarthritis osteophytes and their effect on osteoblasts," Matrix Biology, vol. 62, pp. 75-91, 2017.

[25] T. Auguet, X. Terra, J. A. Porras et al., "Plasma visfatin levels and gene expression in morbidly obese women with associated fatty liver disease," Clinical Biochemistry, vol. 46, no. 3, pp. 202-208, 2013.

[26] Y. H. Lee and S.-C. Bae, "Circulating adiponectin and visfatin levels in rheumatoid arthritis and their correlation with disease activity: a meta-analysis," International Journal of Rheumatic Diseases, vol. 21, no. 3, pp. 664-672, 2018.

[27] R. R. Yammani and R. F. Loeser, "Extracellular nicotinamide phosphoribosyltransferase (NAMPT/visfatin) inhibits insulin-like growth factor-1 signaling and proteoglycan synthesis in human articular chondrocytes," Arthritis Research \& Therapy, vol. 14, no. 1, 2012.

[28] S. Yang, J.-H. Ryu, H. Oh et al., "NAMPT (visfatin), a direct target of hypoxia-inducible factor- $2 \alpha$, is an essential catabolic regulator of osteoarthritis," Annals of the Rheumatic Diseases, vol. 74, no. 3, pp. 595-602, 2015.

[29] A. L. McNulty, M. R. Miller, S. K. O'Connor, and F. Guilak, "The effects of adipokines on cartilage and meniscus catabolism," Connective Tissue Research, vol. 52, no. 6, pp. 523-533, 2011.

[30] Y. Li, X. He, Y. Li et al., "Nicotinamide phosphoribosyltransferase (Nampt) affects the lineage fate determination of mesenchymal stem cells: a possible cause for reduced osteogenesis and increased adipogenesis in older individuals," Journal of Bone and Mineral Research, vol. 26, no. 11, pp. 2656-2664, 2011.

[31] L. Tsiklauri, J. Werner, M. Kampschulte et al., "Visfatin alters the cytokine and matrix-degrading enzyme profile during osteogenic and adipogenic MSC differentiation," Osteoarthritis and Cartilage, vol. 26, no. 9, pp. 1225-1235, 2018.

[32] H. Xie, S.-Y. Tang, X.-H. Luo et al., "Insulin-Like effects of visfatin on human osteoblasts," Calcified Tissue International, vol. 80, no. 3, pp. 201-210, 2007.

[33] S. U. Venkateshaiah, S. Khan, W. Ling et al., "NAMPT/PBEF1 enzymatic activity is indispensable for myeloma cell growth and osteoclast activity," Experimental Hematology, vol. 41, no. 6, pp. 547-557, 2013.

[34] A. Fukuhara, M. Matsuda, M. Nishizawa et al., "Visfatin: a protein secreted by visceral fat that mimics the effects of insulin," Science, vol. 307, no. 5708, pp. 426-430, 2005.

[35] Q. Peng, S. H. Jia, J. Parodo, Y. Ai, and J. C. Marshall, "PreB cell colony enhancing factor induces Nampt-dependent translocation of the insulin receptor out of lipid microdomains in A549 lung epithelial cells," Endocrinology and Metabolism, vol. 308, no. 4, pp. E324-E333, 2015.

[36] W.-S. Huang, C.-N. Chen, C.-I. Sze, and C.-C. Teng, "Visfatin induces stromal cell-derived factor- 1 expression by $\beta 1$ 
integrin signaling in colorectal cancer cells," Journal of Cellular Physiology, vol. 228, no. 5, pp. 1017-1024, 2013.

[37] M. A. Nowell, P. J. Richards, C. A. Fielding et al., "Regulation of pre-B cell colony-enhancing factor by STAT-3-dependent interleukin-6trans-signaling: implications in the pathogenesis of rheumatoid arthritis," Arthritis \& Rheumatism, vol. 54, no. 7, pp. 2084-2095, 2006.

[38] Y. H. Huh, G. Lee, W. H. Song, J. T. Koh, and J. H. Ryu, "Crosstalk between FLS and chondrocytes is regulated by HIF- $2 \alpha$-mediated cytokines in arthritis," Experimental \& Molecular Medicine, vol. 47, 2015.

[39] M. J. Yoon, M. Yoshida, S. Johnson et al., "SIRT1-Mediated eNAMPT secretion from adipose tissue regulates hypothalamic NAD+ and function in mice," Cell Metabolism, vol. 21, no. 5, pp. 706-717, 2015.

[40] E.-H. Hong, H. S. Yun, J. Kim et al., "Nicotinamide phosphoribosyltransferase is essential for interleukin- $1 \beta$-mediated dedifferentiation of articular chondrocytes via SIRT1 and extracellular signal-regulated kinase (ERK) complex signaling," Journal of Biological Chemistry, vol. 286, no. 32, pp. 28619-28631, 2011.

[41] M. Dvir-Ginzberg and J. Steinmeyer, "Towards elucidating the role of SirT1 in osteoarthritis," Frontiers in Bioscience, vol. 18, no. 1, pp. 343-355, 2013.

[42] E.-H. Hong, S.-J. Lee, J.-S. Kim et al., "Ionizing radiation induces cellular senescence of articular chondrocytes via negative regulation of SIRT1 by p38 kinase," Journal of Biological Chemistry, vol. 285, no. 2, pp. 1283-1295, 2010.

[43] S. Liu, S. Qiao, J. Yuan, and D. Liu, "Visfatin stimulates production of monocyte chemotactic protein-1 and interleukin-6 in human vein umbilical endothelial cells," Hormone and Metabolic Research, vol. 41, no. 4, pp. 281-286, 2009.

[44] R. Adya, B. K. Tan, A. Punn, J. Chen, and H. S. Randeva, "Visfatin induces human endothelial VEGF and MMP-2/9 production via MAPK and PI3K/Akt signalling pathways: novel insights into visfatin-induced angiogenesis," Cardiovascular Research, vol. 78, no. 2, pp. 356-365, 2008.

[45] Y. Wang, C. Gao, Y. Zhang et al., "Visfatin stimulates endometrial cancer cell proliferation via activation of PI3K/Akt and MAPK/ERK1/2 signalling pathways," Gynecologic Oncology, vol. 143, no. 1, pp. 168-178, 2016.

[46] J. E. P. Brown, D. J. Onyango, M. Ramanjaneya et al., "Visfatin regulates insulin secretion, insulin receptor signalling and mRNA expression of diabetes-related genes in mouse pancreatic $\beta$-cells," Journal of Molecular Endocrinology, vol. 44, no. 3, pp. 171-178, 2010.

[47] C. Jacques, M. Holzenberger, Z. Mladenovic et al., "Proinflammatory actions of visfatin/nicotinamide phosphoribosyltransferase (nampt) involve regulation of insulin signaling pathway and nampt enzymatic activity," Journal of Biological Chemistry, vol. 287, no. 18, pp. 15100-15108, 2012.

[48] R. J. Buldak, M. Gowarzewski, L. Buldak et al., "Viability and oxidative response of human colorectal HCT-116 cancer cells treated with visfatin/eNampt in vitro," Journal of Physiology and Pharmacology: An Official Journal of the Polish Physiological Society, vol. 4, pp. 557-566, 2015.

[49] A. F. Santidrian, S. E. LeBoeuf, E. D. Wold, M. Ritland, J. S. Forsyth, and B. H. Felding, "Nicotinamide phosphoribosyltransferase can affect metastatic activity and cell adhesive functions by regulating integrins in breast cancer," DNA Repair, vol. 23, pp. 79-87, 2014.

[50] A. von Heideman, A. Berglund, R. Larsson, and P. Nygren, "Safety and efficacy of NAD depleting cancer drugs: results of a phase I clinical trial of CHS 828 and overview of published data," Cancer Chemotherapy and Pharmacology, vol. 65, no. 6, pp. 1165-1172, 2010.

[51] J. A. Khan, X. Tao, and L. Tong, "Molecular basis for the inhibition of human NMPRTase, a novel target for anticancer agents," Nature Structural \& Molecular Biology, vol. 13, no. 7, pp. 582-588, 2006.

[52] N. Busso, M. Karababa, M. Nobile et al., "Pharmacological inhibition of nicotinamide phosphoribosyltransferase/visfatin enzymatic activity identifies a new inflammatory pathway linked to NAD," PloS One, vol. 3, no. 5, 2008.

[53] E. Abdel-Sattar, E. T. Mehanna, S. H. El-Ghaiesh, H. Mohammad, H. A. Elgendy, and S. A. Zaitone, "Pharmacological action of a pregnane glycoside," in Impact on Weight Gain and Energy Expenditure, vol. 9, p. 990, Frontiers in pharmacology, New York, NY, USA, 2018.

[54] Z. Huang, G. Tian, S. Cheng et al., "Polydatin attenuates atherosclerosis in ApoE- $/-$ mice through PBEF mediated reduction of cholesterol deposition," The American Journal of Chinese Medicine, vol. 46, no. 8, pp. 1841-1859, 2018.

[55] C. Tu, D. Gao, X. F. Li et al., "Inflammatory stress potentiates emodin-induced liver injury in rats," Frontiers in Pharmacology, vol. 6, p. 233, 2015.

[56] Z. Qi, S. Qi, L. Ling, J. Lv, and Z. Feng, "Salidroside attenuates inflammatory response via suppressing JAK2-STAT3 pathway activation and preventing STAT3 transfer into nucleus," International Immunopharmacology, vol. 35, pp. 265-271, 2016.

[57] J. Hajavi, A. A. Momtazi, T. P. Johnston, M. Banach, M. Majeed, and A. Sahebkar, "Curcumin: a naturally occurring modulator of adipokines in diabetes," Journal of Cellular Biochemistry, vol. 118, no. 12, pp. 4170-4182, 2017.

[58] C. Li, J. Li, Y. Chen, X. Zhong, and M. Kang, "Effect of curcumin on visfatin and zinc- $\alpha 2$-glycoprotein in a rat model of non-alcoholic fatty liver disease," Acta Cirurgica Brasileira, vol. 31, no. 11, pp. 706-713, 2016.

[59] S.-R. Kim, H.-J. Park, Y.-H. Bae et al., "Curcumin downregulates visfatin expression and inhibits breast cancer cell invasion," Endocrinology, vol. 153, no. 2, pp. 554-563, 2012.

[60] D. Yan, B. He, J. Guo, S. Li, and J. Wang, "Involvement of TLR4 in the protective effect of intra-articular administration of curcumin on rat experimental osteoarthritis," Acta Cirúrgica Brasileira, vol. 34, no. 6, 2019.

[61] J. Wu, M. Lv, and Y. Zhou, "Efficacy and side effect of curcumin for the treatment of osteoarthritis: a meta-analysis of randomized controlled trials," Pakistan Journal of Pharmaceutical Sciences, vol. 1, pp. 43-51, 2019.

[62] H. Cui, S. Li, C. Xu, J. Zhang, Z. Sun, and H. Chen, "Emodin alleviates severe acute pancreatitis-associated acute lung injury by decreasing pre-B-cell colony-enhancing factor expression and promoting polymorphonuclear neutrophil apoptosis," Molecular Medicine Reports, vol. 16, no. 4, pp. 5121-5128, 2017.

[63] Q.-H. Ding, C.-Y. Ye, E.-M. Chen, W. Zhang, and $\mathrm{X} . \mathrm{H}$. Wang, "Emodin ameliorates cartilage degradation in osteoarthritis by inhibiting NF- $\kappa \mathrm{B}$ and $\mathrm{Wnt} / \beta$-catenin signaling in-vitro and in-vivo," International Immunopharmacology, vol. 61, pp. 222-230, 2018.

[64] F. C. Liu, C. C. Wang, J. W. Lu et al., "Chondroprotective effects of genistein against osteoarthritis induced joint inflammation," Nutrients, vol. 11, no. 5, 2019.

[65] Q. Zhu, W. Zhang, D. Mu, H. Zhou, S. Wu, and H. Zou, "Effects of genistein on lipopolysaccharide-induced injury of mouse alveolar epithelial cells and its mechanism," Bioscience, 
Biotechnology, and Biochemistry, vol. 84, no. 3, pp. 544-551, 2020.

[66] J. Yuan, W. Ding, N. Wu, S. Jiang, and W. Li, "Protective effect of genistein on condylar cartilage through downregulating NF- $\kappa \mathrm{B}$ expression in experimentally created osteoarthritis rats," BioMed Research International, vol. 2019, 2019.

[67] J. Hao, C. Chen, K. Huang et al., "Polydatin improves glucose and lipid metabolism in experimental diabetes through activating the Akt signaling pathway," European Journal of Pharmacology, vol. 745, pp. 152-165, 2014.

[68] S. Tang, Q. Tang, J. Jin et al., "Polydatin inhibits the IL-1 $\beta$ induced inflammatory response in human osteoarthritic chondrocytes by activating the Nrf2 signaling pathway and ameliorates murine osteoarthritis," Food \& Function, vol. 9, no. 3, pp. 1701-1712, 2018.

[69] Z. Wu, Z. Luan, X. Zhang et al., "Chondro-protective effects of polydatin in osteoarthritis through its effect on restoring dysregulated autophagy via modulating MAPK, and PI3K/ Akt signaling pathways," Scientific Reports, vol. 1, 2019.

[70] X. Huang, S. Xing, C. Chen, Z. Yu, and J. Chen, "Salidroside protects PC12 cells from A $\beta 1-40$-induced cytotoxicity by regulating the nicotinamide phosphoribosyltransferase signaling pathway," Molecular Medicine Reports, vol. 16, no. 3, pp. 2700-2706, 2017. 